TRANSACTIONS OF THE

AMERICAN MATHEMATICAL SOCIETY

Volume 352, Number 5, Pages 2261-2281

S 0002-9947(99)02527-1

Article electronically published on November 18, 1999

\title{
AN ALGORITHMIC APPROACH TO THE CONSTRUCTION OF HOMOMORPHISMS INDUCED BY MAPS IN HOMOLOGY
}

\author{
MADJID ALLILI AND TOMASZ KACZYNSKI
}

\begin{abstract}
This paper is devoted to giving the theoretical background for an algorithm for computing homomorphisms induced by maps in homology. The principal idea is to insert the graph of a given continuous map $f$ into a graph of a multi-valued representable map $F$. The multi-valued representable maps have well developed continuity properties and admit a finite coding that permits treating them by combinatorial methods. We provide the construction of the homomorphism $F_{*}$ induced by $F$ such that $F_{*}=f_{*}$. The presented construction does not require subsequent barycentric subdivisions and simplicial approximations of $f$. The main motivation for this paper comes from the project of computing the Conley Index for discrete dynamical systems.
\end{abstract}

\section{INTRODUCTION}

Recent applications of the Conley Index theory and the fixed point theory to the study of chaos in dynamical systems [17, 18, 19, 20, 24, 25] can be characterized by a marriage of the abstract algebraic topology with rigorous computer assisted numerics. Thus the progress in that area generates a lot of interest in algorithms for computing structures of algebraic topology and in particular, homology of spaces and maps. The computation of topological structures has already been applied to such areas as: graph coloring, image processing, cartography, computer graphics, solid modeling, mesh generation, molecular modeling. We refer to the survey paper [5] for the discussion of the raising branch that authors propose to call Computational Topology.

The computation of homology is a very fresh topic since only recently effective algorithms and their complexity estimations started to appear; see e.g. [3, 4] 9]. In the applications discussed in [5, low dimensional topological structures are sufficient to study and, in fact, the most efficient algorithms such as 3 are only valid for homology of a polyhedron in $\mathbb{R}^{3}$. However, applications of homology theory in analysis and dynamical systems require computation in the space of an arbitrary dimension, and not just homology of spaces but primarily homology of maps. This is a partial achievement of [12] which provides an efficient reduction algorithm for computing homology of finitely generated chain complexes (of any dimension) and, simultaneously, of a homomorphism induced in homology by a chain map. However the difficulty is not in computing the homology of a chain map but in computing a chain map induced by a continuous map, and this is exactly what is addressed

Received by the editors June 2, 1997 and, in revised form, January 14, 1998.

1991 Mathematics Subject Classification. Primary 55-04; Secondary 54C60, 54H20, 05 B25.

Key words and phrases. Algorithm, homology, representable map, Vietoris map.

The second author was supported by grants from NSERC of Canada and FCAR of Quebec. 
in this paper. To the authors' best knowledge, there are no general results yet on computing homology of continuous maps. Several attempts have been made to compute the fixed point index or a topological degree but programs fail in all but the most simple cases of maps such as complex polynomials addressed in [14]. The authors of this paper believe that the lack of success is mainly due to two reasons: one may be related to insufficient skills in algorithm design and programming among mathematicians interested in the topic and to a communication barrier between topologists and computer scientists. That communication problem is addressed by the authors of [5] and we hope that our work responds to their appeal. A second reason is that computing a chain map induced by a continuous map by definition involves simplicial approximations and fine barycentric subdivisions. That is a very heavy tool which leads to involved computations even in the most simple examples which should be computable by hand.

This paper provides an algorithmic construction of such homomorphisms based on an entirely different idea than simplicial approximations. In Section 2 we define a class $\mathcal{R}$ of representable polyhedra in $\mathbb{R}^{n}$ first introduced by Mischaikow and Mrozek [18, 19, 20] for a given convex grid $\mathcal{E}$, and classes of perfect and almost perfect representable maps which are multi-valued maps whose graphs are representable polyhedra in the product space. A reader without a strong background in algebraic topology may ignore the discussion of a CW complex structure defined by $\mathcal{E}$ and assume that $\mathcal{E}$ is a cubic grid as in Example 2.2(ii). A perfect representation of a continuous map $f$ in $\mathbb{R}^{n}$ is a representable map $F: X \longrightarrow Y, X, Y \in \mathcal{R}$ with convex values which is fully determined by its values on vertices of a given grid and whose graph contains the graph of $f$, whereas an almost perfect representation has star-shaped values and it is fully determined by its values on the cells of the highest dimension. We give examples in Section 6 to illustrate these concepts. The formula that permits computing the homology of an almost perfect map is the same as for a perfect map but we present both types of maps since opinions on which of the two constructions is more useful and natural are divided.

In Section 3 we give an explicit construction of the chain map $\varphi: C(X) \longrightarrow$ $C(Y)$ for $F$ such that $F_{*}$ can be defined as the quotient map on the homology groups $\varphi_{*}: H(X) \longrightarrow H(Y)$. We prove in Section 4 that our definition agrees with the classical definition of $F_{*}$ based on the Vietoris-Begle Theorem [2 22] and hence it coincides with $f_{*}: H(X) \longrightarrow H(Y)$. Thus the main results of this paper are described by the following theorems:

Theorem 3.1. Let $\mathcal{E}$ be a convex grid in $\mathbb{R}^{n}, X$ and $Y$ representable sets over $\mathcal{E}$ and $F: X \longrightarrow \mathcal{P}(Y)$ a perfect (respectively almost perfect) map over $\mathcal{E}(X)$. Then, there exists a chain map $\varphi: C(X) \longrightarrow C(Y)$ such that

$$
\left|\varphi_{q} \sigma\right| \subset \mathcal{A}(\sigma) \quad \forall \sigma \in \mathcal{E}^{q}(X)
$$

The chain map $\varphi$ is called an $\mathcal{E}$-chain map induced by $F$.

Corollary 4.7. Let $F: X \longrightarrow Y$ be a perfect (respectively an almost perfect) map and $\varphi: C(X) \longrightarrow C(Y)$ any chain map given by Theorem 3.1. Then $\varphi_{*}=F_{*}$.

Corollary 4.8. Let $f: X \longrightarrow \mathbb{R}^{n}$ be a single-valued continuous map, $X \in \mathcal{R}$, and let $F: X \longrightarrow \mathbb{R}^{n}$ be a perfect (respectively an almost perfect) representation of $f$. 
Let $Y \in \mathcal{R}$ be a representable set with $f(X) \subset F(X) \subset Y$, and let $\varphi: C(X) \longrightarrow$ $C(Y)$ be any chain map given for $F$ by Theorem [3.1. Then $\varphi_{*}=f_{*}$, where $f$ is considered as a map from $X$ to $Y$.

It is also directly proved in Section 3 that any two chain maps given by Theorem 3.1 are chain homotopic, so they induce the same homomorphism in homology (Theorem 3.3), and that the construction is preserved under subdivisions of the grid (Theorem 3.7).

The inductive proof of Theorem 3.1 together with the discussion of a co-boundary construction following it, serves as the base of an algorithm for computing $\varphi$ which is in progress [1, 13]. The idea of our construction originates from [22]; however, the important asset is that, in our case, there are no barycentric subdivisions and subsequent chain-approximations required.

Although the background idea reaches the old Vietoris-Begle Theorem 2, as far as computing is of concern, this seems to be a completely new and promising approach to that topic. Moreover, this approach is very appealing to computer scientists working on image processing: Indeed, in the planar case, a graph on a computer screen obtained by a drawing tool is a chain of small painted grid squares whose sizes depend on the computer capacity and screen resolution. Therefore it is a perfect representation of the exact graph contained inside as defined in Section 2

The fact that we avoided barycentric subdivisions being crucial for the definition of $f_{*}$ in the classical approach (see [6] 21]) may seem unbelievable and, in fact, there is a minor difficulty hidden in our statement: if the input information consists of a pair of polyhedra $X, Y$ and a continuous map $f: X \longrightarrow Y$, then one must be aware of the fact that the representation of $f$ is a star-shape valued map $F$ : $X \longrightarrow \mathbb{R}^{n}$ whose range is not necessarily in $Y$. Namely, $F(X)$ is a finite covering of $f(X) \subset Y$ by star-shaped representable polyhedra in $\mathbb{R}^{n}$, not in $Y$. This difficulty is overcomed in Section 5 as follows:

One may pass to a subdivision $\mathcal{E}^{\prime}$ of $\mathcal{E}$ (defined independently of $f$ ) such that $Y$ is a strong deformation retract of the $\mathcal{E}^{\prime}$-representable star collar $Z=\operatorname{st}_{\mathcal{E}^{\prime}}(Y)$. In case of a cubic grid, it is enough to subdivide each edge to three equal parts. In case of a triangulation, one may take the second barycentric subdivision. Then an $\mathcal{E}^{\prime}$-representation $F: X \longrightarrow Z$ is computed as well as the isomorphism $r_{*}$ : $H(Z) \longrightarrow H(Y)$ induced by the deformation. Finally one defines $f_{*}=r_{*} \circ F_{*}$.

It should be noted that, in many applications to analysis and dynamical systems, the described above passage to a subdivision of the grid will not be necessary. Indeed, we will often consider a continuous map $f: \mathbb{R}^{n} \longrightarrow \mathbb{R}^{n}$, its appropriate representation $F: \mathbb{R}^{n} \longrightarrow \mathbb{R}^{n}$, and the spaces $X$ and $Y$ to which the domain and codomain of both maps should be restricted will be fixed a posteriori. That is the case in the computation of the Conley Index which is the main motivation for this paper. The definition and properties of the Conley Index for discrete dynamical systems are too involved to present them in this introduction, therefore we refer the reader to the literature [10, 16, 18, 19, 23 for details justifying the following comment: In the computation of the Conley Index of an isolated invariant set $S$ of a map $f$, via its multivalued representation $F$, the only place where a grid subdivision may appear necessary is when making a guess of so called isolating neighborhood for F. In [23], Szymczak provides a simple algorithm for checking 
if a given representable set $N$ is indeed an isolating neighborhood. Once that is confirmed, the remaining computations are performed in a fixed grid.

\section{Discretization of SPACE AND REPRESEntAble MAPS}

Definition 2.1. $\mathcal{E} \subset \mathcal{P}\left(\mathbb{R}^{n}\right)$ is called a convex grid in $\mathbb{R}^{n}$ if the following conditions are satisfied:

1. $\mathbb{R}^{n}=\bigcup_{e \in \mathcal{E}} e$.

2. $e \neq e^{\prime} \Longrightarrow e \cap e^{\prime}=\emptyset$.

3. $e \in \mathcal{E} \Longrightarrow e$ is a convex polyhedron without boundary.

4. $B \subset \mathbb{R}^{n}$ bounded $\Longrightarrow \operatorname{st} B:=\{e \in \mathcal{E}: \bar{e} \cap \bar{B} \neq \emptyset\}$ is finite.

- If $e^{\prime}$ is a face of $e$, we write $e^{\prime} \leq e$.

\section{Example 2.2.}

(i) Any simplicial triangulation.

(ii) The cubic grid $\mathcal{E}_{k}$ of mesh $\frac{1}{k}, k \in \mathbb{N}^{*}$

$$
\begin{gathered}
e_{k}=\frac{1}{k}(x+Q), \text { where } x \in \mathbb{Z}^{n}, \\
\left.Q=I_{1} \times I_{2} \times \ldots \times I_{n} \text { and } I_{j}=\right] 0,1[,\{0\} \text { or }\{1\} .
\end{gathered}
$$

Let $X \subset \mathbb{R}^{n}$ and $\mathcal{E}$ a convex grid. The set $X$ is said to be representable over $\mathcal{E}$ if there exists $\mathcal{E}^{\prime} \subset \mathcal{E}$ such that $\mathcal{E}^{\prime}$ is finite and $X=\bigcup_{e \in \mathcal{E}^{\prime}} e=\bar{X}$. $\mathcal{E}^{\prime}$ is then denoted by $\mathcal{E}(X)$. We will also use the notations $\mathcal{E}^{q}(X)$ for the set of all cells of dimension $q$ in $X\left(\mathcal{E}^{0}(X)\right.$ is the set of all vertices of $\mathcal{E}$ in $\left.X\right)$ and $\mathcal{R}$ for the family of all representable polyhedra in $\mathbb{R}^{n}$ over $\mathcal{E}$.

The notation $\cos _{\mathcal{E}} B$ will stand for the smallest convex set which is a union of cells of $\mathcal{E}$ and containing $B \subset \mathbb{R}^{n}$. In general, such union may be infinite (it might happen that $\cos _{\mathcal{E}} B=\mathbb{R}^{n}$ ) even if $B$ is bounded. We therefore add the following axiom to the definition of the convex grid

5. $\cos _{\mathcal{E}} B \in \mathcal{R}$ if $B$ is bounded.

This axiom is obviously satisfied in the previous examples. If $\mathcal{E}$ is a cubic grid, then $\operatorname{co}_{\mathcal{E}} B$ is a product of intervals.

Given a representable set $X$ in $\mathbb{R}^{n}$, the grid $\mathcal{E}(X)$ defines on $X$ the structure of a CW complex with the pasting maps of $q$-cells $e \in \mathcal{E}^{q}(X)$ simply being the inclusions of the geometric boundary bd(e) into the $(q-1)$-skeleton $X^{q-1}=$ $\bigcup\left\{\sigma: \quad \sigma \in \mathcal{E}^{i}(X), \quad i=0,1, \ldots, q-1\right\}$ of $X$. We recall from [15, Ch. IX that the group of $q$-chains of $X, C_{q}(X)=H_{q}\left(X^{q}, X^{q-1}\right)$ is the direct sum of $H_{q}(\bar{e}, \operatorname{bd}(e)) \approx \mathbb{Z}$ over $e \in \mathcal{E}^{q}(X)$, thus $C_{q}(X)$ may be identified with the free Abelian group generated by $\mathcal{E}^{q}(X)$. For the general definition of the boundary homomorphism $\partial_{q}: C_{q}(X) \longrightarrow C_{q-1}(X)$ defining the chain complex structure on $C(X)=\left\{C_{q}(X)\right\}_{q \in \mathbb{Z}}$, we refer to [15], Ch. IX. However, we shall give the explicit definition in the case of a cubic grid $\mathcal{E}_{k}$ described in Example 2.2 (ii) which is our primary interest.

Clearly, it is sufficient to present the definition when $k=1$. First, for the cell $e=I^{q}=[0,1]^{q} \subset \mathbb{R}^{q}$, we put

$$
A_{i} e=\left\{\left(x_{1}, \ldots, x_{i-1}, 0, x_{i+1}, \ldots, x_{q}\right): \quad x \in I^{q}\right\}
$$


and

$$
B_{i} e=\left\{\left(x_{1}, \ldots, x_{i-1}, 1, x_{i+1}, \ldots, x_{q}\right): \quad x \in I^{q}\right\} .
$$

Following [15], Ch. VII, $A_{i} e$ and $B_{i} e$ are called, respectively, the front $i$-face and the back $i$-face of $e$. Now any $q$-cell $e \in \mathcal{E}^{q}(X), X \subset \mathbb{R}^{n}$ is the image of $I^{q}$ under the affine linear map composed of an insertion of zeros on chosen $(n-q)$ coordinates with the translation of the origin to a vertex $x \in \mathbb{Z}^{n}$. We put $A_{i} e=T\left(A_{i} I^{q}\right), \quad B_{i} e=T\left(B_{i} I^{q}\right)$ and let

$$
\partial_{q} e=\sum_{i=1}^{q}(-1)^{i}\left(A_{i} e-B_{i} e\right)
$$

For the verification of the property $\partial \circ \partial=0$, we again refer to [15], Ch. VII. A somewhat similar construction is also given in [7].

A map $F: X \longrightarrow \mathcal{P}(Y)$, where $X$ and $Y$ are two arbitrary sets will be called a multi-valued map from $X$ to $Y$. For $A \subset X$ and $B \subset Y$, we denote by

$$
\begin{gathered}
\operatorname{dom} F=\{x \in X: F(x) \neq \emptyset\}, \\
F(A)=\bigcup_{x \in A} F(x),
\end{gathered}
$$

respectively, the domain of $F$ and the image of $F$.

Assume $X$ and $Y$ to be metric spaces. $F$ is upper semi-continuous (u.s.c.) if $\{x \in \operatorname{dom} F: F(x) \subset U\}$ is open for any open subset $U$ of $Y$. Equivalently, $\{x \in \operatorname{dom} F: F(x) \cap A \neq \emptyset\}$ is closed for any closed subset $A$ of $Y$.

Definition 2.3. Let $F: \operatorname{dom} F \subset X \longrightarrow \mathcal{P}(Y)$ be a multi-valued mapping such that $\operatorname{dom} F \in \mathcal{R}$ and $F(x)$ is representable for each $x$ in $X$. We say that $F$ is representable over $\mathcal{E}(X)$ if

a) $F(x)=F(y) \quad \forall x, y \in e \in \mathcal{E}(X)$;

b) $G(F)=\overline{G(F)}$, where $G(F)$ denotes the graph of $F$.

We can observe that a) and b) $\Longrightarrow\left(e^{\prime} \leq e \Longrightarrow F(e) \subseteq F\left(e^{\prime}\right)\right)$.

In what follows, we introduce the notions of perfect and almost perfect representations of a continuous map with a representable domain in $\mathbb{R}^{n}$ and indicate ways to construct them.

Definition 2.4. Let $\mathcal{E}$ be a convex grid in $\mathbb{R}^{n}, X \in \mathcal{R}$ and $F: X \longrightarrow \mathcal{R}$ be a multivalued representable map over $\mathcal{E}(X)$ with $\operatorname{dom} F=X$. F is said to be perfect over $\mathcal{E}(X)$ if

i) $F(x)$ is convex and closed $\forall x \in X$;

ii) $F(e)=\bigcap\left\{F(v): v \leq e, v \in \mathcal{E}^{0}(X)\right\} \forall e \in \mathcal{E}(X)$;

iii) $\mathcal{A}(v)=\bigcap\{F(\gamma): v \leq \gamma, \gamma \in \mathcal{E}(X)\} \neq \emptyset \forall v \in \mathcal{E}^{0}(X)$.

Remark 2.5.

1) It is obviously seen that $F(e) \subset \bigcap\left\{F(v): v \leq e, v \in \mathcal{E}^{0}(X)\right\}$.

2) The property iii) of the definition above implies

$$
\mathcal{A}(\sigma)=\bigcap\{F(\gamma): \sigma \leq \gamma, \gamma \in \mathcal{E}(X)\} \neq \emptyset \quad \forall \sigma \in \mathcal{E}(X) .
$$


Lemma 2.6. Let $F$ be a representable map over $\mathcal{E}(X)$ with convex and closed values. Then $F$ has a continuous selector $f$ if and only if

$$
\mathcal{A}(v)=\bigcap\{F(\gamma): v \leq \gamma, \gamma \in \mathcal{E}(X)\} \neq \emptyset \quad \forall v \in \mathcal{E}^{0}(X) .
$$

Proof. Indeed, if $f$ is a continuous selector of $F$ and $v \in \mathcal{E}^{0}(X)$, then $f(v) \in F(e)$ for any $e \in \mathcal{E}(X)$ such that $v \leq e$. Therefore $f(v) \in \mathcal{A}(v)$. Conversely, let condition (2.1) be satisfied. Then we can define $f$ on vertices by choosing $f(v) \in \mathcal{A}(v)$ for all $v \in \mathcal{E}^{0}(v)$. Since $\mathcal{A}(v) \neq \emptyset$ and $F$ has convex closed values, the definition of $f$ can be extended to convex cells by linearity to obtain a continuous selector of $F$.

Let $\mathcal{E}$ be a convex grid in $\mathbb{R}^{n}, X \in \mathcal{R}$. A cell $\sigma \in \mathcal{E}(X)$ is called primitive (in $X)$ if there exists no $\tau \in \mathcal{E}(X)$ such that $\sigma$ is a proper face of $\tau$.

Definition 2.7. A representable multivalued map $F: X \longrightarrow \mathcal{R}$ with $\operatorname{dom} F=X$, is called almost perfect over $\mathcal{E}(X)$ if the following axioms are satisfied:

i) $F(\sigma)$ is convex for each primitive cell $\sigma \in \mathcal{E}(X)$;

ii) $F(e)=\bigcup\{F(\sigma): e \leq \sigma, \sigma$ primitive in $X\} \forall e \in \mathcal{E}(X)$;

iii) $\mathcal{A}(v) \neq \emptyset$ for each vertex $v \in \mathcal{E}^{0}(X)$.

It is easy to see that $\mathcal{A}(e)=\bigcap\{F(\sigma): e \leq \sigma, \sigma$ primitive in $X\}$. Therefore, $\mathcal{A}(e)$ is a convex set for each $e \in \mathcal{E}(X)$. Moreover $\mathcal{A}(e) \neq \emptyset$ by iii) and $\mathcal{A}(e) \subset$ $F(e)$. This implies that $F(e)$ is star-shaped for each $e \in \mathcal{E}(X)$. Obviously, each perfect map is almost perfect. Conversely, if $F$ is an almost perfect map, then $G$ given by $G(x)=\operatorname{co}_{\mathcal{E}} F(x)$ is perfect. Here is the philosophy behind those two definitions. Perfect maps are naturally constructed by defining their values on vertices in $\mathcal{E}^{0}(X)$ and extending the definition to cells of higher dimension by intersections. Almost perfect maps are constructed in the reverse order from the cells of highest dimension down to cells of dimension zero by unions.

Let $X \in \mathcal{R}$ and a continuous single valued map $f: X \longrightarrow \mathbb{R}^{n}$. A perfect (respectively almost perfect) representation of $f$ is any perfect (respectively almost perfect) map $F: X \longrightarrow \mathcal{R}$ such that $f(x) \in F(x)$ for all $x \in X$. It is seen that in order to construct a perfect (respectively almost perfect) representation of $f$, the following numerical information is sufficient.

1) Sufficiently good approximations of $f$ at finitely many points of $X$ : the vertices in $\mathcal{E}^{0}(X)$ for a perfect map, and some interior points (e.g. mid-points) of primitive cells in $X$ for an almost perfect map.

2) The modulus of uniform continuity of $f$ on $X$, i.e. the function $\varepsilon \longrightarrow \delta_{\varepsilon}$ such that

$$
\|x-y\|<\delta_{\varepsilon} \Longrightarrow\|f(x)-f(y)\|<\varepsilon,
$$

for each $x, y \in X$.

Moreover, by taking sufficiently fine grids, one can obtain a representation with values of arbitrarily small diameters. Indeed, in order to make the above discussion precise, let $\mathcal{E}_{k}$ be the $k$-th cubic grid described at the beginning of the section. We choose the norm $\|x\|=\max \left\{\left|x_{i}\right|: i=1,2, \ldots, n\right\}$ in $\mathbb{R}^{n}$ so that the balls $B_{r}(x)=\{y:\|y-x\|<r\}$ are $n$-dimensional cubes.

Let $\varepsilon \longrightarrow \delta_{\varepsilon}$ be the modulus of uniform continuity of $f: X \longrightarrow \mathbb{R}^{n}$ with respect to that norm. Given $\varepsilon>0$, let $k \in \mathbb{N}$ be such $\frac{1}{k}<\min \left\{\delta_{\varepsilon}, \varepsilon\right\}$. Let $x_{\sigma}$ be the 
center of each primitive cell $\sigma \in \mathcal{E}_{k}(X)$ and let $y_{\sigma}$ be an $\varepsilon$-approximation of $f\left(x_{\sigma}\right)$. By the assumption on $k$, we may find $y_{\sigma} \in \mathcal{E}^{0}(X)$. For each $x \in \bar{\sigma}$ we have

$$
\left\|f(x)-y_{\sigma}\right\| \leq\left\|f(x)-f\left(x_{\sigma}\right)\right\|+\left\|f\left(x_{\sigma}\right)-y_{\sigma}\right\|<2 \varepsilon .
$$

Let $m \in \mathbb{N}$ be such that $\frac{m}{k} \geq 2 \varepsilon$. We may define

$$
F(x)=F(\sigma):=\bar{B}_{\frac{m}{k}}\left(y_{\sigma}\right), \quad \forall x \in \sigma,
$$

for each primitive cell $\sigma$. By the above inequality, we have $f(x) \in F(x)$ for each $x \in \bar{\sigma}$ and the values of $F$ have diameters $\frac{4 m}{k}$ which can be made close to $8 \varepsilon$ if $k$ is big enough. The same construction can be done for perfect maps.

\section{HOMOMORPHISM INDUCED IN HOMOLOGY BY PERFECT AND ALMOST PERFECT MAPS}

Let $\mathcal{E}$ be a convex grid in $\mathbb{R}^{n}$. For $X \in \mathcal{R}$, we denote by $C_{q}(X)$ the $q$-dimensional chain group of the chain complex $C(X)$ generated by $\mathcal{E}^{q}(X)$.

Given $\sigma=\sum_{i} c_{i} e_{i} \in C_{q}(X)$, we denote the carrier of $\sigma$ by $|\sigma|=\bigcup\left\{e_{i}: c_{i} \neq 0\right\}$.

In the sequel, we construct a chain map $\varphi: C(X) \longrightarrow C(Y)$ such that

$$
\varphi_{*}=F_{*}: H(X) \longrightarrow H(Y) \text {. }
$$

Theorem 3.1. Let $\mathcal{E}$ be a convex grid in $\mathbb{R}^{n}, X$ and $Y$ representable sets over $\mathcal{E}$ and $F: X \longrightarrow \mathcal{P}(Y)$ a perfect (respectively almost perfect) map over $\mathcal{E}(X)$. Then, there exists a chain map $\varphi: C(X) \longrightarrow C(Y)$ such that

$$
\left|\varphi_{q} \sigma\right| \subset \mathcal{A}(\sigma) \quad \forall \sigma \in \mathcal{E}^{q}(X) .
$$

The chain map $\varphi$ is called an $\mathcal{E}$-chain map induced by $F$.

Proof. By induction, we construct $\varphi_{q}: C_{q}(X) \longrightarrow C_{q}(Y)$ such that

$$
\left|\varphi_{q} \sigma\right| \subset \mathcal{A}(\sigma)=\bigcap\{F(\gamma): \sigma \leq \gamma, \gamma \in \mathcal{E}(X)\} \quad \forall \sigma \in C_{q}(X) .
$$

For $q=0$ and $q=1$ : Let $v$ be a vertex. Since $\mathcal{A}(v) \neq \emptyset$, we can choose a vertex $\varphi_{0}(v) \in \mathcal{A}(v)$.

For $\sigma \in \mathcal{E}^{1}(X)$ such that $\partial_{1} \sigma=v_{1}-v_{0}$, we put $z=\varphi_{0}\left(\partial_{1} \sigma\right)=\varphi_{0}\left(v_{1}\right)-\varphi_{0}\left(v_{0}\right)$. It is easily seen that $|z| \subset \mathcal{A}(\sigma)$.

Let $\varepsilon: C_{0}(X) \longrightarrow \mathbb{Z}$ be the augmentation map for $C_{0}(X)$. Since $\varphi_{0}$ is an augmentation preserving, it follows that $\varepsilon(z)=0$. Now as $\mathcal{A}(\sigma)$ is a convex set, $\tilde{H}_{0}(\mathcal{A}(\sigma))$ is trivial and then there exists a 1-chain $c \in C_{1}(\mathcal{A}(\sigma))$ such that $\partial_{1} c=z$. We then define

$$
\varphi_{1} \sigma:=c .
$$

It is easily seen that condition (3.1) is automatically satisfied and the boundary operator $\partial$ satisfies by definition

$$
\varphi_{0} \partial_{1}(\sigma)=\partial_{1} \varphi_{1}(\sigma) .
$$

Suppose now that $\varphi_{i}$ is constructed for $0 \leq i<q$ where $\varphi_{i}: C_{i}(X) \longrightarrow C_{i}(Y)$ satisfies the conditions

$$
\begin{gathered}
\left|\varphi_{i} \sigma\right| \subset \mathcal{A}(\sigma) \quad \forall \sigma \in \mathcal{E}^{i}(X), \\
\varphi_{i-1} \partial=\partial \varphi_{i} \quad \text { for } \quad 1 \leq i \leq q-1 .
\end{gathered}
$$


Construction of $\varphi_{q}$ : Let $\sigma \in \mathcal{E}^{q}(X)$. Then, $\partial \sigma=\sum_{s} c_{s} \sigma_{s}$, where $\sigma_{s} \in \mathcal{E}^{q-1}(X) \forall s$.

We have $\left|\varphi_{q-1} \sigma_{s}\right| \subset \mathcal{A}\left(\sigma_{s}\right) \subset \mathcal{A}(\sigma) \forall s$. Thus,

$$
\left|\varphi_{q-1} \partial \sigma\right| \subset \mathcal{A}(\sigma) \text {. }
$$

Since $\mathcal{A}(\sigma)$ is convex, and $\varphi_{q-1} \partial \sigma$ is a cycle (by the inductive assumption), there exists a chain $c \in C_{q}(\mathcal{A}(\sigma))$ such that $\partial c=\varphi_{q-1} \partial \sigma$. We then define

$$
\varphi_{q} \sigma:=c .
$$

By definition, $\varphi_{q}$ satisfies the following assumptions:

$\partial \varphi_{q}=\varphi_{q-1} \partial$.

If $\sigma \in \mathcal{E}^{q}(X)$, then $\varphi_{q} \sigma \in C_{q}(\mathcal{A}(\sigma))$ and then $\left|\varphi_{q} \sigma\right| \subset \mathcal{A}(\sigma)$.

3.1. Construction of a coboundary of a given cycle. The above proof contains a statement on the existence of a chain $c$ such that $\partial c=\varphi(\partial \sigma)$ which seems, at first sight, to be an obstruction to a precise algorithm. We discuss below a geometric construction of a coboundary $c$ of a given cycle $z$, i.e. a chain $c$ such that $\partial c=z$, in the case of a cubic grid. When $\operatorname{dim} z=1$, the main idea comes from A. Szymczak (personal communication). The detailed proof of the correctness of our construction and a formal algorithm will be presented in [1, 13].

Let $\mathcal{E}=\mathcal{E}_{1}$ be the unitary cubic grid in $\mathbb{R}^{n}$ and $A \subset \mathbb{R}^{n}$ a representable rectangle. Given $z \in Z_{q}(A)$, we construct a chain $c \in C_{q+1}(A)$ such that $\partial_{q+1} c=$ $z$. We denote the procedure by $c:=C O B(z)$ where $C O B$ stands for the word coboundary.

We may write

$$
z=\sum_{i=1}^{N} c_{i} e_{i} \quad \text { where } \quad e_{i} \in \mathcal{E}^{q}(A) .
$$

Each $e_{i}$ can be expressed in the form

$$
e_{i}=\left(\left(a_{i}\right)_{1},\left(b_{i}\right)_{1}\right) \times \ldots \times\left(\left(a_{i}\right)_{n},\left(b_{i}\right)_{n}\right)
$$

where

$$
(a, b)=\left\{\begin{array}{lll}
] a, b[ & \text { if } & a \neq b, \\
\{a\} & \text { if } & a=b .
\end{array}\right.
$$

The endpoints of intervals $\left(a_{i}\right)_{k},\left(b_{i}\right)_{k}$ are integers, either $\left(b_{i}\right)_{k}=\left(a_{i}\right)_{k}+1$ or $\left(b_{i}\right)_{k}=\left(a_{i}\right)_{k}$, and the first equality holds exactly for $q$ values of $k$.

Let $R(z)$ be the smallest rectangle in $A$ containing $|z|$ (the carrier of $z$ ). We have

$$
R(z)=\left[m_{1}, M_{1}\right] \times\left[m_{2}, M_{2}\right] \times \ldots \times\left[m_{n}, M_{n}\right]
$$

where $m_{k}=\min _{i}\left\{\left(a_{i}\right)_{k}\right\}$ and $M_{k}=\max _{i}\left\{\left(b_{i}\right)_{k}\right\}$.

Let $k_{1}$ be the first nontrivial coordinate of $R(z)$, i.e. the smallest integer $k$ with the property $m_{k} \neq M_{k}$,

$$
R_{k_{1}}(z)=\left\{x \in R(z): x_{k_{1}}=m_{k_{1}}\right\},
$$

and

$$
P_{k_{1}}: R(z) \longrightarrow R_{k_{1}}(z)
$$


be the canonical projection. Let

$$
\pi_{q}: C_{q}(R(z)) \longrightarrow C_{q}\left(R_{k_{1}}(z)\right)
$$

be the homomorphism induced by $P_{k_{1}}$. $\pi_{q}$ is given on elementary cubes $e \in R(z)$ as follows:

$$
\pi_{q}(e)= \begin{cases}0 & \text { if } b_{k_{1}}=a_{k_{1}}+1, \\ \left(a_{1}, b_{1}\right) \times \ldots \times\left(a_{k_{1}-1}, b_{k_{1}-1}\right) \times\left\{m_{k_{1}}\right\} & \\ \times\left(a_{k_{1}+1}, b_{k_{1}+1}\right) \times \ldots \times\left(a_{n}, b_{n}\right) & \text { if } b_{k_{1}}=a_{k_{1}},\end{cases}
$$

where $e=\left(a_{1}, b_{1}\right) \times \ldots \times\left(a_{n}, b_{n}\right)$.

We construct $\operatorname{COB}(z)$ by induction with respect to $d$, the dimension of $R(z)$. Obviously the dimension of $R(z)$ is at least $q$ so the induction starts from $d=q$.

If $d=q$, then $C_{q+1}(R(z))=0$ which implies that $B_{q}(R(z))=0$ and since $\tilde{H}_{q}(R(z))=0, Z_{q}(R(z))=0$. As $z \in Z_{q}(R(z))$, therefore $z=0$. We put $c:=$ $C O B(z)=0$.

Suppose the construction is done for dimensions up to a certain $d \geq q$ and now let $\operatorname{dim} R(z)=d+1$. For $e=\left(a_{1}, b_{1}\right) \times \ldots \times\left(a_{n}, b_{n}\right)$, define

$$
\left[\pi_{q}(e), e\right]= \begin{cases}\sum_{i=m_{k_{1}}}^{0}\left\{\left(a_{1}, b_{1}\right) \times \ldots \times\left(a_{k_{1}-1}, b_{k_{1}-1}\right) \times(i, i+1)\right. & \text { if } \pi_{q}(e)=0 \\ \left.\times\left(a_{k_{1}+1}, b_{k_{1}+1}\right) \times \ldots \times\left(a_{n}, b_{n}\right)\right\} & \text { otherwise }\end{cases}
$$

Note that $\left|\left[\pi_{q}(e), e\right]\right|$ is a $q+1$-dimensional rectangle through which $\pi_{q}(e)$ is lifted up to $e$.

$C O B\left(\pi_{q}(z)\right)$ is defined by induction hypothesis, since $\pi_{q}(z)$ is a cycle contained in $R_{k_{1}}(z)$ which is a rectangle of dimension $d$.

Define

$$
C O B(z):=\operatorname{COB}\left(\pi_{q}(z)\right)+\sum_{i} c_{i}\left[\pi_{q}\left(e_{i}\right), e_{i}\right] .
$$

By using the fact that $\partial z=0$, it can be verified that

$$
\partial\left(\sum_{i} c_{i}\left[\pi_{q}\left(e_{i}\right), e_{i}\right]\right)=z-\pi_{q}(z)
$$

By induction hypothesis $\partial C O B\left(\pi_{q}(z)\right)=\pi_{q}(z)$. Therefore

$$
\partial C O B(z)=\pi_{q}(z)+z-\pi_{q}(z)=z .
$$

We illustrate this construction by the following example.

Example 3.2. Consider the clockwise oriented cycle

$$
z=e_{8}+e_{7}+e_{6}+e_{5}-e_{4}-e_{3}-e_{2}-e_{1}
$$

in the plane where $e_{i}$ are positively oriented intervals indicated in Figure1. Note that $z \in C_{1}(R(z))$ where $R(z)=[0,2] \times[0,2]$.

We have $k_{1}=1, R_{1}(z)=\{0\} \times[0,2]$, and $\pi_{1}(z)=0$. It is easily seen that

$$
\left[\pi_{1}\left(e_{1}\right), e_{1}\right]=\left[\pi_{1}\left(e_{2}\right), e_{2}\right]=\left[\pi_{1}\left(e_{7}\right), e_{7}\right]=\left[\pi_{1}\left(e_{5}\right), e_{5}\right]=0
$$




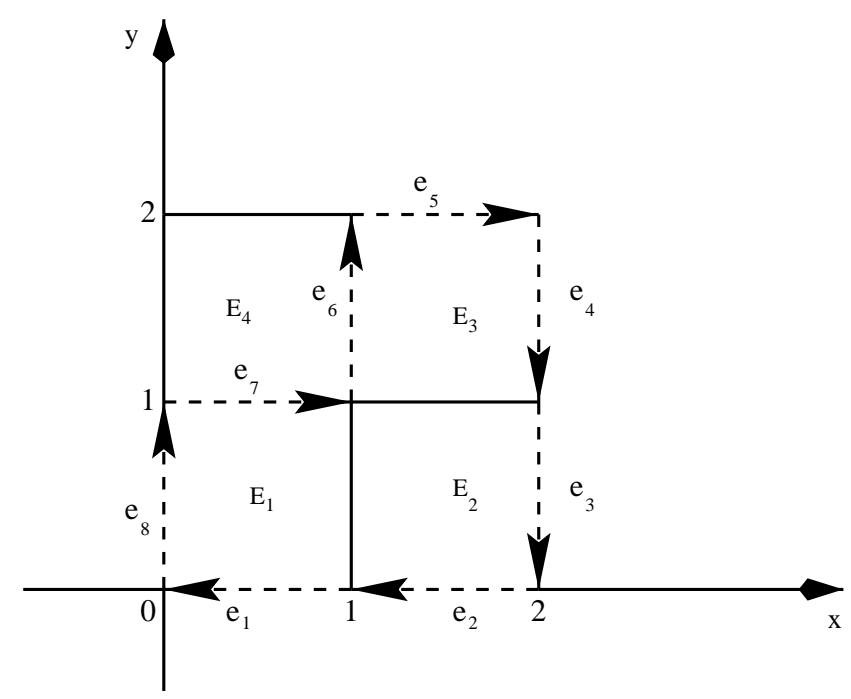

FiguRE 1. Coboundary construction

$$
\left[\pi_{1}\left(e_{8}\right), e_{8}\right]=0, \quad\left[\pi_{1}\left(e_{6}\right), e_{6}\right]=E_{4}, \quad\left[\pi_{1}\left(e_{4}\right), e_{4}\right]=E_{3}+E_{4}
$$

and

$$
\left[\pi_{1}\left(e_{3}\right), e_{3}\right]=E_{1}+E_{2},
$$

where $E_{1} \ldots E_{4}$ are positively oriented squares as indicated in Figure 1. Hence

$$
\begin{aligned}
C O B(z) & =C O B\left(\pi_{1}(z)\right)+0+0+E_{4}+0-E_{4}-E_{3}-E_{1}-E_{2} \\
& =-\left(E_{1}+E_{2}+E_{3}\right) .
\end{aligned}
$$

The following results will establish that the homomorphism $\varphi_{*}: H_{*}(X) \longrightarrow$ $H_{*}(Y)$ induced by $F$ in homology is independent of the choice of the chain map induced by $F$, given by Theorem 3.1 and invariant with respect to subdivisions of the original grid.

Theorem 3.3. Suppose that $\varphi$ and $\psi$ are two $\mathcal{E}$-chain maps induced by the same perfect (respectively almost perfect) map $F$. Then $\varphi$ and $\psi$ are chain homotopic.

Proof. By induction, we construct $D_{q}: C_{q}(X) \longrightarrow C_{q+1}(Y)$ such that

$$
\left|D_{q} \sigma\right| \subset \mathcal{A}(\sigma)=\bigcap\{F(\gamma): \sigma \leq \gamma, \gamma \in \mathcal{E}(X)\} \quad \forall \sigma \in C_{q}(X) .
$$

For $q=0$ : Let $v$ be a vertex. Since $\mathcal{A}(v)$ is convex, $\varphi_{0}(v), \psi_{0}(v) \in \mathcal{A}(v)$ and $\overline{H_{1}(\mathcal{A}(v))}=0$, there exists a 1 -chain $c_{1} \in C_{1}(\mathcal{A}(v))$ such that

$$
\partial c_{1}=\varphi_{0}(v)-\psi_{0}(v) \text {. }
$$

Note that $\varphi_{0}(v)-\psi_{0}(v)$ is a cycle.

We then define $D_{0} v:=c_{1}$.

$D_{0}: C_{0}(X) \longrightarrow C_{1}(Y)$ is well defined and satisfies:

$\left|D_{0} v\right| \subset \mathcal{A}(v) \forall v \in \mathcal{E}^{0}(X)$;

$\partial_{1} D_{0}=\varphi_{0}-\psi_{0}$.

Suppose now that $D_{i}$ is constructed for $0 \leq i<q$ where $D_{i}: C_{i}(X) \longrightarrow C_{i+1}(Y)$ satisfies the conditions: 


$$
\begin{aligned}
& \left|D_{i} \sigma\right| \subset \mathcal{A}(\sigma) \forall \sigma \in \mathcal{E}^{i}(X) ; \\
& \partial_{i+1} D_{i}+D_{i-1} \partial_{i}=\varphi_{i}-\psi_{i} \quad \text { for } \quad 0 \leq i \leq q-1 .
\end{aligned}
$$

Construction of $D_{q}$ :

Let $\sigma \in \mathcal{E}^{q}(X)$. Then $\partial \sigma=\sum_{s} c_{s} \sigma_{s}$, where $\sigma_{s} \in \mathcal{E}^{q-1}(X) \forall s$. Set

$$
\alpha=\varphi_{q} \sigma-\psi_{q} \sigma-D_{q-1} \partial \sigma .
$$

We have $\left|\varphi_{q} \sigma\right| \cup\left|\psi_{q} \sigma\right| \subset \mathcal{A}(\sigma)$ and $\left|D_{q-1} \sigma_{s}\right| \subset \mathcal{A}\left(\sigma_{s}\right) \subset \mathcal{A}(\sigma) \forall \sigma_{s}$. Thus $\left|D_{q-1} \partial \sigma\right| \subset \mathcal{A}(\sigma)$ and consequently $|\alpha| \subset \mathcal{A}(\sigma)$. $\alpha$ is a cycle. Indeed,

$$
\partial_{q} \alpha=\partial_{q} \varphi_{q} \sigma-\partial_{q} \psi_{q} \sigma-\partial_{q} D_{q-1} \partial_{q} \sigma=\varphi_{q-1} \partial_{q} \sigma-\psi_{q-1} \delta_{q} \sigma-\partial_{q} D_{q-1} \partial_{q} \sigma .
$$

Since, by the inductive assumptions,

$$
\varphi_{q-1} \partial_{q} \sigma-\psi_{q-1} \partial_{q} \sigma=\partial_{q} D_{q-1} \partial_{q} \sigma+D_{q-2} \partial_{q-1} \partial_{q}
$$

we deduce that $\partial_{q} \alpha=0$.

Since $\mathcal{A}(\sigma)$ is convex, and $\alpha$ is a cycle, there exits a $(q+1)$-chain $c_{q+1} \in$ $C_{q+1}(\mathcal{A}(\sigma))$ such that $\partial_{q+1} c_{q+1}=\alpha$. We then define

$$
D_{q} \sigma:=c_{q+1} \text {. }
$$

By definition, $D_{q}$ satisfies the following assumptions:

$$
\begin{aligned}
& \left|D_{q} \sigma\right| \subset \mathcal{A}(\sigma) ; \\
& \partial_{q+1} D_{q}+D_{q-1} \partial_{q}=\varphi_{q}-\psi_{q} .
\end{aligned}
$$

Proposition 3.4. Let $F$ and $G$ be perfect multivalued maps over $\mathcal{E}(X)$ such that $F(X) \subset G(X)$ for all $x \in X$. Then any $\mathcal{E}$-chain map induced by $F$ is also an $\mathcal{E}$-chain map induced by $G$.

Proof. If $\varphi$ is an $\mathcal{E}$-chain map induced by $F$, then it obviously satisfies the property

$$
\begin{aligned}
\left|\varphi_{q} e\right| & \subset \bigcap\{F(\sigma): e \leq \sigma, \sigma \in \mathcal{E}(X)\} \\
& \subset \bigcap\{G(\sigma): e \leq \sigma, \sigma \in \mathcal{E}(X)\}, \quad \forall e \in \mathcal{E}^{q}(X) .
\end{aligned}
$$

Thus, $\varphi$ is an $\mathcal{E}$-chain map induced by $G$.

Definition 3.5. We say that a grid $\mathcal{E}^{\prime}$ is a subgrid of a grid $\mathcal{E}$ if each cell in $\mathcal{E}$ is a representable set with respect to $\mathcal{E}^{\prime}$.

Remark 3.6. In the case of cubic grids it is obvious that $\mathcal{E}^{\prime}=\mathcal{E}_{m}$ is a subgrid of $\mathcal{E}=\mathcal{E}_{k}$ if and only if $k$ divides $m$.

Theorem 3.7. Let $\mathcal{E}^{\prime}$ be a subgrid of $\mathcal{E}$. If $F: X \longrightarrow \mathcal{P}(Y)$ is a perfect map over $\mathcal{E}(X)$, then $F$ is a perfect map over $\mathcal{E}^{\prime}(X)$ and it induces the same homomorphism in homology.

Proof of the theorem. To prove the theorem we shall first prove the following lemmas.

Lemma 3.8. Let $v$ be a vertex of the subgrid $\mathcal{E}^{\prime}$ of $\mathcal{E}$ and $F$ be a perfect map over $\mathcal{E}$. Then we have either of the two situations:

1) If $v$ is a vertex of the original grid $\mathcal{E}$, then

$$
\begin{aligned}
\mathcal{A}_{\mathcal{E}}(v) & =\bigcap\{F(\sigma): v \leq \sigma, \sigma \in \mathcal{E}(X)\}=\mathcal{A}_{\mathcal{E}^{\prime}}(v) \\
& =\bigcap\left\{F\left(\sigma^{\prime}\right): v \leq \sigma, \sigma^{\prime} \in \mathcal{E}^{\prime}(X)\right\} .
\end{aligned}
$$


2) If not, then there exists a unique cell $e \in \mathcal{E}(X)$ such that $v \in$ inte and

$$
\mathcal{A}_{\mathcal{E}^{\prime}}(v)=\mathcal{A}_{\mathcal{E}}(e) .
$$

Proof.

1) The proof is immediate if we observe that each cell of $\mathcal{E}^{\prime}$ having $v$ as a vertex is contained in some cell of $\mathcal{E}$ having $v$ as a vertex and conversely each cell of $\mathcal{E}$ having $v$ as a vertex contains some cell of $\mathcal{E}^{\prime}$ having $v$ as a vertex.

2) Observe that each cell of $\mathcal{E}$ having $e$ as a face contains some cell of $\mathcal{E}^{\prime}$ having $v$ as a vertex and each cell of $\mathcal{E}^{\prime}$ having $v$ as a vertex is contained in some cell of $\mathcal{E}$ having $e$ as a face.

Lemma 3.9. If $F$ is perfect over $\mathcal{E}$, then $F$ is perfect over $\mathcal{E}^{\prime}$.

Proof. Since $\operatorname{dom} F \in \mathcal{R}_{\mathcal{E}}$ and $\forall x \in \operatorname{dom} F, F(x) \in \mathcal{R}_{\mathcal{E}}$ and since each cell of $\mathcal{E}$ is a finite union of cells of $\mathcal{E}^{\prime}$, we deduce that $\operatorname{dom} F \in \mathcal{R}_{\mathcal{E}^{\prime}}$ and $F(x) \in \mathcal{R}_{\mathcal{E}^{\prime}} \forall x$. The equality $G(F)=\overline{G(F)}$ is obvious. Let $e^{\prime} \in \mathcal{E}^{\prime}$. There exists $e \in \mathcal{E}$ such that $e^{\prime} \subset e$. Since $F(x)=F(y) \quad \forall x, y \in e$, the relation $F(x)=F(y)$ remains true for all $x, y \in e^{\prime}$. Thus $F$ is representable over $\mathcal{E}^{\prime}$.

$F(x)$ is obviously convex and closed for all $x \in \operatorname{dom} F$. For $e^{\prime} \in \mathcal{E}^{\prime}$, we can easily see that

$$
F\left(e^{\prime}\right)=\bigcup\left\{F(v): v \leq e^{\prime}, v \in\left(\mathcal{E}^{\prime}\right)^{0}\right\} .
$$

We first can observe that the inclusion

$$
F\left(e^{\prime}\right) \subset \bigcap\left\{F(v): v \leq e^{\prime}, v \in\left(\mathcal{E}^{\prime}\right)^{0}\right\}
$$

is obviously satisfied. We will prove the other inclusion by distinguishing two cases:

1) There exists $v_{0} \leq e^{\prime}$ and $e \in \mathcal{E}$ such that $v_{0} \in \operatorname{int}(e)$ and $e^{\prime} \subset e$. Then,

$$
\bigcap\left\{F(v): v \leq e^{\prime}, v \in\left(\mathcal{E}^{\prime}\right)^{0}\right\} \subset F\left(v_{0}\right)=F(e)=F\left(e^{\prime}\right) .
$$

2) If not, $e^{\prime} \in \mathcal{E}$ and each vertex of $e^{\prime}$ is a vertex of the original grid. Then the precedent equality follows immediately.

Let $v \in\left(\mathcal{E}^{\prime}\right)^{0}$. If there exists some $e \in \mathcal{E}$ such that $v \in \operatorname{int}(e)$, then by the precedent lemma $\mathcal{A}_{\mathcal{E}^{\prime}}(v)=\mathcal{A}_{\mathcal{E}}(e) \neq \emptyset$. Else, $v$ is a vertex of the original grid and again by the precedent lemma $\mathcal{A}_{\mathcal{E}^{\prime}}(v)=\mathcal{A}_{\mathcal{E}}(v) \neq \emptyset$.

We denote by $\mu: C(X, \mathcal{E}) \longrightarrow C\left(X, \mathcal{E}^{\prime}\right)$ the first chain derivation on $X$, i.e. the homomorphism satisfying the following properties:

1) $\mu_{0}: C_{0}(X, \mathcal{E}) \longrightarrow C_{0}\left(X, \mathcal{E}^{\prime}\right)$ is the inclusion map, i.e. $\mu_{0}(v)=v, \quad \forall v \in$ $\mathcal{E}^{0}(X)$, since each vertex of $\mathcal{E}$ is again a vertex of $\mathcal{E}^{\prime}$.

2) For a $p$-cell $\sigma \in \mathcal{E}^{p}(X)$,

$$
\mu_{p}(\sigma)=\sum_{i} \sigma_{i}
$$

where $\sigma_{i}$ is a $p$-simplex of $\mathcal{E}^{\prime}(X)$ contained in $\sigma$ and having the same orientation as $\sigma$.

Theorem 3.1 applied to $F$ defined on $(X, \mathcal{E})$ and respectively $\left(X, \mathcal{E}^{\prime}\right)$ yields two chain maps $\varphi$ and $\psi$ induced by $F$.

We will construct by induction a chain homotopy $D$ between $\varphi: C(X, \mathcal{E}) \longrightarrow$ $C(Y)$ and $\psi \circ \mu: C(X, \mathcal{E}) \longrightarrow C(Y)$. 
Let $v \in \mathcal{E}^{0}(X)$. Recall that $\mathcal{A}_{\mathcal{E}}(v)=\mathcal{A}_{\mathcal{E}}^{\prime}(v), \varphi_{0}(v) \in \mathcal{A}_{\mathcal{E}}(v), \psi_{0}(v) \in \mathcal{A}_{\mathcal{E}^{\prime}}(v)$ and $\mu_{0}(v)=v$. Since $\varphi_{0}(v)-\phi_{0}(v) \in C_{0}\left(\mathcal{A}_{\mathcal{E}}(v)\right)$ is a cycle and $\mathcal{A}_{\mathcal{E}}(v)$ is convex, there exists a 1-chain $c_{1} \in C_{1}\left(\mathcal{A}_{\mathcal{E}}(v)\right)$ such that $\partial_{1} c_{1}=\varphi_{0}(v)-\psi_{0}(v)$. We then define $D_{0}(v):=c_{1} . D_{0}$ satisfies:

1) $\left|D_{0}(v)\right| \subset \mathcal{A}_{\mathcal{E}}(v)=\mathcal{A}_{\mathcal{E}}^{\prime}(v)$

2) $\partial_{1} D_{0}(v)=\partial_{1} c_{1}=\varphi_{0}(v)-\psi_{0} \circ \mu_{0}(v)$.

Suppose that for $0 \leq i \leq q-1$ we have the existence of a chain map $D_{i}$ : $C_{i}(X) \longrightarrow C_{i+1}(Y)$ such that

1) $\left|D_{i}(\sigma)\right| \subset \mathcal{A}_{\mathcal{E}}(\sigma)$;

2) $\partial_{i+1} D_{i}(\sigma)+D_{i-1} \partial_{i}(\sigma)=\varphi_{i}(\sigma)-\psi_{i} \circ \mu_{i}(\sigma)$.

Let $\sigma \in \mathcal{E}^{q}(X)$. We know that $\mu_{q}(\sigma)=\sum_{s} \sigma_{s}$, where $\sigma_{s} \in\left(\mathcal{E}^{\prime}\right)^{q}(X), \sigma_{s} \subset \sigma$ and $\sigma_{s}$ has the same orientation as $\sigma$. Then consider

$$
\alpha=\varphi_{q}(\sigma)-\psi_{q} \circ \mu_{q}(\sigma)-D_{q-1} \partial_{q}(\sigma) .
$$

It is easily seen that

$$
\left|\psi_{q}\left(\sigma_{s}\right)\right| \subset \mathcal{A}_{\mathcal{E}}^{\prime}\left(\sigma_{s}\right) \subset \mathcal{A}_{\mathcal{E}}(\sigma)
$$

and since $\partial_{q} \sigma=\sum_{i} \sigma_{i}$, where $\sigma_{i} \in \mathcal{E}^{(q-1)}(X), \mathcal{A}_{\mathcal{E}}\left(\sigma_{i}\right) \subset \mathcal{A}_{\mathcal{E}}(\sigma)$ and by the inductive assumptions, we deduce that $D_{q-1} \partial_{q} \sigma \subset \mathcal{A}_{\mathcal{E}}(\sigma)$. Thus, $|\alpha| \subset \mathcal{A}_{\mathcal{E}}(\sigma)$. It is easily proven that $\alpha$ is a cycle and since $\alpha \in C_{q}\left(\mathcal{A}_{\mathcal{E}}(\sigma)\right)$, where $\mathcal{A}_{\mathcal{E}}(\sigma)$ is convex, there exists a $(q+1)$-chain $c_{q+1} \in C_{q+1}\left(\mathcal{A}_{\mathcal{E}}(\sigma)\right)$ such that $\partial_{q+1} c_{q+1}=\alpha$. We then define $D_{q}(\sigma):=c_{q+1}$.

$D_{q}: C_{q}(X) \longrightarrow C_{q+1}(Y)$ satisfies all the inductive hypotheses.

Remark 3.10. All the above results can be extended to almost perfect maps with a slight modification of the arguments in the proof of Lemma 3.8

We can observe that the proof of Theorem [3.7] is independent on the subgrid of $\mathcal{E}$ choosen on $Y$.

By combining Proposition 3.4 and Theorem 3.7 one obtains the following.

Corollary 3.11. If $F$ is a perfect (respectively almost perfect) map with respect to a grid $\mathcal{E}$ and $G$ is a selection of $F$, i.e. $G(x) \subset F(x) \forall x$, which is perfect (respectively almost perfect) with respect to a subgrid $\mathcal{E}^{\prime}$ of $\mathcal{E}$, then $G$ and $F$ induce the same homomorphism in homology.

\section{Product Grids and Vietoris-Begle Theorem}

In this section, it is proved that the construction of the chain map $\varphi$ provided by Theorem 3.1 agrees with the classical definitions of homomorphisms induced in homology by single-valued and multivalued maps.

Definition 4.1. Let $\mathcal{E}=\mathcal{E}(X)$ and $\mathcal{E}^{\prime}=\mathcal{E}(Y)$ be convex grids respectively in $X$ and $Y$. Then,

$$
\mathcal{E} \times \mathcal{E}^{\prime}:=\left\{e \times e^{\prime}: e \in \mathcal{E}, \quad e^{\prime} \in \mathcal{E}^{\prime}\right\}
$$

is a convex grid in $X \times Y$ called the product of $\mathcal{E}$ and $\mathcal{E}^{\prime}$ with

$$
\left(\mathcal{E} \times \mathcal{E}^{\prime}\right)^{n}:=\left\{e \times e^{\prime}: \operatorname{dim} e+\operatorname{dim} e^{\prime}=n\right\}
$$

being the basis of the $n$-dimensional chain group $C_{n}(X \times Y)$. (See [ $[$ ].) 
It is easily proved that

Proposition 4.2. Let $F$ be a representable map over $\mathcal{E}$ and $\mathcal{E}^{\prime}$ then $G(F)$ is a representable set over the convex grid $\mathcal{E} \times \mathcal{E}^{\prime}$.

The chain maps $p_{\#}: C(G(F)) \longrightarrow C(X)$ and $q_{\#}: C(G(F)) \longrightarrow C(Y)$ induced by the projections $p: G(F) \longrightarrow X$ and $q: G(F) \longrightarrow Y$ are given by

$$
p_{\#}\left(e \times e^{\prime}\right)= \begin{cases}0 & \text { if } \operatorname{dim} e<n, \\ e & \text { if } \operatorname{dim} e=n\end{cases}
$$

and

$$
q_{\#}\left(e \times e^{\prime}\right)= \begin{cases}0 & \text { if } \operatorname{dim} e^{\prime}<n, \\ e^{\prime} & \text { if } \operatorname{dim} e^{\prime}=n .\end{cases}
$$

Let $X$ and $Y$ be compact and Hausdorff topological spaces and $f: X \longrightarrow Y$ be a continuous map. We recall from [8] that $f$ is called a Vietoris map if

1. $f$ is onto;

2. $f^{-1}(y)$ is acyclic $\forall y \in Y$.

We have the following classical result.

Theorem 4.3 (Vietoris-Begle Theorem, [2]). If $f: X \longrightarrow Y$ is a Vietoris map, then $f_{*}: \check{H}(X) \longrightarrow \check{H}(Y)$ is an isomorphism, where $\check{H}$ denotes the Čech homology with field coefficients.

Remark 4.4. The Vietoris-Begle Theorem, in its general formulation, holds for Vietoris or Čech homologies with coefficients in a field. In this paper, however, we apply it in the context of finite polyhedra and maps $f$ with polyhedral inverse images $f^{-1}(y)$, where the theorem holds true for any homology theory and any ring of coefficients.

Definition 4.5 (See [6]). Let $F: X \longrightarrow Y$ be a multivalued acyclic and uppersemi-continuous map and $p, q$ the canonical projections from $G(F)$ onto (respectively) $X$ and $Y$. Then $p: G(F) \longrightarrow X$ is a Vietoris map. Thus $p_{*}: H(G(F)) \longrightarrow$ $H(X)$ is an isomorphism. The homomorphism

$$
F_{*}: H(X) \longrightarrow H(Y)
$$

is defined by the formula

$$
F_{*}=q_{*} \circ\left(p_{*}\right)^{-1} \text {. }
$$

The following theorem provides the link between the above definition and the construction of $\varphi$ in Section 3 .

Theorem 4.6. Let $F$ be a perfect (respectively an almost perfect) map. Then there exists a chain map $\psi: C(X) \longrightarrow C(G(F))$ such that

$$
|\psi(\sigma)| \subseteq \bar{\sigma} \times \mathcal{A}(\sigma) \quad \forall \sigma \in \mathcal{E}(X) .
$$

Moreover, $\varphi=q_{\#} \circ \psi: C(X) \longrightarrow C(Y)$ satisfies the conclusions of Theorem 3.1 and $\psi$ is the homotopic inverse of the chain map $p_{\#}$.

$\mathbf{1}$ - Construction of $\psi$. We proceed by induction.

For a vertex $v$ in $X$, we set $\psi_{0}(v)=v \times v_{0}$, where $v_{0}$ is any vertex of $\mathcal{A}(v)$. 
Suppose that for $0 \leq i \leq q-1$, there exists a homomorphism $\psi_{i}: C_{i}(X) \longrightarrow$ $C_{i}(G(F))$ such that

$$
\left\{\begin{array}{l}
\left|\psi_{i}(\sigma)\right| \subseteq \bar{\sigma} \times \mathcal{A}(\sigma) \quad \forall \sigma \in \mathcal{E}^{i}(X) \\
\psi_{i-1} \partial=\partial \psi_{i}
\end{array}\right.
$$

Let $\sigma \in \mathcal{E}^{q}(X)$. Since $\psi_{q-1}(\partial \sigma)$ is a cycle, $\left|\psi_{q-1}(\partial \sigma)\right| \subseteq \bar{\sigma} \times \mathcal{A}(\sigma)$ and $\bar{\sigma} \times \mathcal{A}(\sigma)$ is convex, there exists $c \in \bar{\sigma} \times \mathcal{A}(\sigma)$ such that $\partial c=\psi_{q-1}(\partial \sigma)$. Then, we define

$$
\psi_{q}(\sigma):=c
$$

It is now straightforward that $q_{\#} \circ \psi$ satisfies all the hypotheses of Theorem 3.1 $\mathbf{2}-\psi$ is a chain homotopic inverse of the chain map $p_{\#}$. We first prove by induction that $p_{\#} \circ \psi=i d_{C(X)}$.

Let $v$ be a vertex of $X$. Then

$$
p_{\#} \circ \psi(v)=p_{\#}\left(v \times v_{0}\right)=v=i d_{C(X)}(v) .
$$

Suppose that for $0 \leq i \leq q-1$ we have $p_{\#} \circ \psi=i d_{C(X)}$ and now let $i=q$.

Given $\sigma \in \mathcal{E}^{q}(X)(\sigma \neq \emptyset)$, we have

$$
p_{\#_{q-1}} \circ \psi_{q-1}\left(\partial_{q} \sigma\right)=\partial_{q} \sigma=\partial_{q}\left[p_{\#_{q}} \circ \psi(\sigma)\right]
$$

and by definition of $p_{\#}$ and $\psi,\left|p_{\# q} \circ \psi(\sigma)\right| \subset \bar{\sigma}$. Then $p_{\#} \circ \psi_{q}(\sigma)$ is a nontrivial $q$-chain which has the same boundary as $\sigma$ and since its carrier is contained in $\bar{\sigma}$ it follows that $p_{\#_{q}} \circ \psi_{q}(\sigma)=\sigma$.

We now construct by induction a chain homotopy between $\psi \circ p_{\#}$ and $i d_{C(G(F))}$.

For a vertex $v \times v^{\prime}$ of $G(F)$, we have $\psi_{0} \circ p_{\#_{0}}\left(v \times v^{\prime}\right)=\psi_{0}(v)=v \times v_{0}, v^{\prime} \in F(v)$ and $v_{0} \in \mathcal{A}(v) \subset F(v)$. Thus,

$$
\left|\psi_{0} \circ p_{\#_{0}}\left(v \times v^{\prime}\right)-i d_{C(G(F))}\left(v \times v^{\prime}\right)\right| \subseteq v \times F(v) .
$$

Since $v \times F(v)$ is star-shaped, there exists $c \in C_{1}(v \times F(v))$ such that

$$
\partial c=\psi_{0} \circ p_{\#_{0}}\left(v \times v^{\prime}\right)-i d_{C(G(F))}\left(v \times v^{\prime}\right) .
$$

We then define

$$
D_{0}\left(v \times v^{\prime}\right):=c .
$$

Suppose that for $0 \leq i \leq q-1$, we have $D_{i}: C_{i}(G(F)) \longrightarrow C_{i+1}(G(F))$ such that

1) $\partial_{i+1} \circ D_{i}+D_{i-1} \circ \partial_{i}=\psi \circ p_{\#}-i d_{C(G(F))}$;

2) $\left|D_{i}\left(e \times e^{\prime}\right)\right| \subseteq \mathcal{F}(e)=\bigcup\{\sigma \times F(\sigma): \sigma \leq e\}=p^{-1}(\bar{e})$.

Let $y_{0} \in F(e)$. It can be verified that the convex set $\bar{e} \times y_{0}$ is a deformation retract of $\mathcal{F}(e)$, hence $\mathcal{F}(e)$ is acyclic. In the case when $F$ is a perfect map, the deformation

$$
H: \mathcal{F}(e) \times[0,1] \longrightarrow \mathcal{F}(e),
$$

can be explicitly given by

$$
H((x, y), t)=\left(x, t y_{o}+(1-t) y\right)
$$

since $F(\sigma)$ is convex with $F(e) \subset F(\sigma)$ for all $\sigma \leq e$.

Now let $i=q$. Given $e \times e^{\prime} \in\left(\mathcal{E} \times \mathcal{E}^{\prime}\right)^{q}[G(F)]$, we set

$$
\alpha=\psi \circ p_{\#}\left(e \times e^{\prime}\right)-i d_{C_{*}(G(F))}\left(e \times e^{\prime}\right)-D_{q-1} \partial_{q}\left(e \times e^{\prime}\right) .
$$


It is easily seen that $\left|\psi \circ p_{\#}\left(e \times e^{\prime}\right)\right| \subset \bar{e} \times \mathcal{A}(e) \subset \mathcal{F}(e)$ and $\left|e \times e^{\prime}\right| \subset \bar{e} \times F(e) \subset$ $\mathcal{F}(e)$.

Lying on the two precedent inductive assumptions, we can see that $|\alpha| \subseteq \mathcal{F}(e)$.

Since $\alpha$ is a cycle in $\mathcal{F}(e)$ and $\mathcal{F}(e)$ is acyclic, there exists $c \in C_{q+1}(\mathcal{F}(e))$ such that $\partial c=\alpha$. We then set

$$
D_{q}\left(e \times e^{\prime}\right):=c
$$

The conclusion is now straightforward.

Corollary 4.7. Let $F: X \longrightarrow Y$ be a perfect (respectively an almost perfect) map and $\varphi: C(X) \longrightarrow C(Y)$ any chain map given by Theorem [3.1. Then $\varphi_{*}=F_{*}$.

Proof. The conclusion follows instantly from Theorem 4.6 and Theorem 3.3

Corollary 4.8. Let $f: X \longrightarrow \mathbb{R}^{n}$ be a single-valued continuous map, $X \in \mathcal{R}$, and let $F: X \longrightarrow \mathbb{R}^{n}$ be a perfect (respectively an almost perfect) representation of $f$. Let $Y \in \mathcal{R}$ be a representable set with $f(X) \subset F(X) \subset Y$, and let $\varphi: C(X) \longrightarrow$ $C(Y)$ any chain map given for $F$ by Theorem 3.1 Then $\varphi_{*}=f_{*}$, where $f$ is considered as a map from $X$ to $Y$.

Proof. Let $j: G(f) \longrightarrow G(F)$ be the embedding map and let $p: G(F) \longrightarrow X$, $q: G(F) \longrightarrow Y$ be the previously discussed projections. We know that $p_{*}$ : $H(G(F)) \longrightarrow H(X)$ is an isomorphism but, on the other hand, $p \circ j: G(f) \longrightarrow X$ is a homeomorphism, therefore, $(p \circ j)_{*}: H(G(f)) \longrightarrow H(X)$ is an isomorphism. Thus, $j_{*}: H(G(f)) \longrightarrow H(G(F))$ is an isomorphism, and we get

$$
f_{*}=\left((q \circ j) \circ(p \circ j)^{-1}\right)_{*}=q_{*} \circ j_{*} \circ j_{*}^{-1} \circ\left(p_{*}\right)^{-1}=F_{*} .
$$

As discussed in the introduction, the aim of the above theorems is providing an explicit construction of the homomorphism $f_{*}$ for a single-valued $f$ via its multivalued representation $F$. Thus $F$ has a continuous selection by the definition, and the assumptions that $F$ is a perfect or an almost perfect map are very natural. However, it follows from Vietoris-Begle Theorem that the homomorphism $F_{*}$ is well defined even if $F$ does not have any continuous selection. It is therefore of interest to provide an explicit construction of $F_{*}$ for a representable map $F$ possibly without a continuous selection. This is achieved in the following.

Theorem 4.9. Let $F: X \longrightarrow \mathbb{R}^{n}$ be a representable map with nonempty convex values. Then, there exists a chain map $\psi: C(X) \longrightarrow C(G(F))$ with the following properties:

a) $|\psi(\sigma)| \subseteq p^{-1}(\bar{\sigma}) \forall \sigma \in \mathcal{E}(X)$;

b) $\psi_{*}=\left(p_{*}\right)^{-1}$.

Moreover, $\varphi=q_{\#} \circ \psi: C(X) \longrightarrow C(Y)$ satisfies $\varphi(\sigma) \subset F(\bar{\sigma}) \quad \forall \sigma \in \mathcal{E}(X)$ and $\varphi_{*}=F_{*}$.

Proof. The set $p^{-1}(\bar{\sigma})$ is acyclic. Indeed, since $F(\sigma)$ is convex for each $\sigma$ and $F(\sigma) \subset F(\tau)$ whenever $\tau \leq \sigma$, the acyclic set $\bar{\sigma} \times y_{0}$ is a deformation retract of $p^{-1}(\bar{\sigma})$ for any $y_{0} \in F(\sigma)$, with the same homotopy as defined in the proof of Theorem 4.6. Therefore we may repeat the arguments of that proof with $\bar{\sigma} \times \mathcal{A}(\sigma)$ replaced throughout by $p^{-1}(\bar{\sigma})$. 
Remark 4.10. It is visible that the conditions of convexity could be replaced by some acyclicity conditions throughout the paper. We purposely avoided that generality since the verification that a given representable set is acyclic, is a task of a large computational complexity, whereas it is given for granted in the case of cubic grids and either perfect maps sending vertices to representable rectangles or almost perfect maps sending primitive cells to such sets. However, a reader interested in a more general setting can easily notice the following: The theorems of Section 3 hold true with the same proofs for the class of representable maps $F: X \longrightarrow Y$ satisfying the following condition.

(A) For each $e \in \mathcal{E}(X), F(e)$ and $F(\bar{e})$ are acyclic.

The theorems of this section hold true for representable maps satisfying

$\left(\mathrm{A}^{\prime}\right)$ For each $e \in \mathcal{E}(X)$, the subsets $p^{-1}(e)$ and $p^{-1}(\bar{e})$ are acyclic.

\section{HOMOMORPHISM INDUCED IN HOMOLOGY BY A CONTINUOUS MAP}

Let $X, Y \in \mathbb{R}^{n}$ be representable with respect to a given grid $\mathcal{E}$ and let $f$ : $X \longrightarrow Y$ be a continuous map. The goal of this paper is a construction of the homomorphism $f_{*}: H(X) \longrightarrow H(Y)$. If $f$ admits a perfect or almost perfect representation $F$ with $F(X) \subset Y$, the answer already is provided by Corollary 4.7 and by the construction of $\varphi_{*}=F_{*}$ in Section 3. In general, that is not guaranteed and we outline the construction that does not rely on the assumption $F(X) \subset Y$.

For simplicity, consider the cubic grids $\mathcal{E}_{k}$ defined in Example 2.2(ii). Let $\mathcal{E}=$ $\mathcal{E}_{1}$ and let $X, Y$ be representable with respect to $\mathcal{E}$. We set

$$
Z=\operatorname{st}_{\mathcal{E}_{k}} Y:=\bigcup\left\{e \in \mathcal{E}_{k}: \bar{e} \cap Y \neq \emptyset\right\} .
$$

We have the following.

Theorem 5.1. If $k \geq 3$, then $Y$ is a strong deformation retract of $Z$. Consequently, the inclusion $i: Y \longrightarrow Z$ induces an isomorphism in homology.

The detailed proof will be presented in [1,13] but we shall provide here its outline which also indicates how to construct the homological inverse of $i$, i.e. a chain map $r: C(Z) \longrightarrow C(Y)$ such that

$$
r_{*}=i_{*}{ }^{-1}: H(Z) \longrightarrow H(Y) .
$$

As a matter of fact, it is the above formula which is important for the construction of $f_{*}$. Suppose that a cell $s \in \mathcal{E}_{k}(Z) \backslash \mathcal{E}_{k}(Y)$ is an exterior face of $Z$, i.e. $s$ is a proper face of an exact one cell $\sigma \in \mathcal{E}_{k}(Z)$ and, moreover, $\sigma \notin \mathcal{E}_{k}(Y)$. Then one may apply the exterior face collapsing described in [12] which gives the homomorphism

$$
r: C(Z) \longrightarrow C(Z \backslash(\sigma \cup s))
$$

defined on generators by

$$
r(e)= \begin{cases}e & \text { if } e \neq s \text { and } e \neq \sigma, \\ 0 & \text { if } e=\sigma \\ s-c \partial \sigma & \text { if } e=s\end{cases}
$$

where $c=+1$ or -1 is the coefficient of $s$ in the expansion of $\partial \sigma$.

It is verified in [12] that $r$ is the homological inverse of the inclusion

$$
i: C(Z \backslash(\sigma \cup s)) \subset C(Z) \text {. }
$$


Then $Z$ is replaced by $Z \backslash \sigma$ and the procedure is repeated by recurrence as long as there remain pairs of cells $(s, \sigma)$ with the above described property. By the assumption $k \geq 3$, it can be proved that, if there are no such pairs left, then $Z=Y$.

Let now $F: X \rightarrow \mathbb{R}^{n}$ be a perfect or almost perfect representation of $f$ with respect to $\mathcal{E}_{k}$ with values of diameter less than or equal to $\frac{1}{k}$, where $k \geq 3$. Then $F(X) \subset Z$. It remains to define

$$
f_{*}:=r_{*} \circ F_{*}
$$

where $F_{*}: H(X) \longrightarrow H(Z)$ and $r_{*}: H(Z) \longrightarrow H(Y)$ are previously discussed.

Remark 5.2. If a simplicial triangulation $\mathcal{E}$ of $\mathbb{R}^{n}$ is considered instead of a cubical grid, Theorem 5.1 holds true with $\mathcal{E}_{k}, k \geq 3$, replaced by the second barycentric subdivision of $\mathcal{E}$.

\section{ExAmples}

In this section, we give two simple examples illustrating the concept of almost perfect and perfect maps. Since the continuous map $f$ whose representation is constructed already is a simplicial map, it is obvious that we cannot bring here any improvement with respect to the technique of simplicial approximations but we rather provide a double check. The true examples of applications to nonlinear maps must involve numerical computations, and the computer programs are still in progress.

Example 6.1. Let $X=\partial\left([0,1]^{2}\right)$, i.e., $X$ is the boundary of the cartesian product $[0,1] \times[0,1]$. We parametrize $X$ by $P:[0,+\infty[\longrightarrow X, \quad(x, y)=P(t)$, where $t$ is the arclength gained by travelling from $P(0)=(0,0)$ to $(x, y)$ counterclockwise.

Let $f: X \longrightarrow X$ be the doubling map defined by $f(P(t))=P(2 t), \quad t \in[0,4]$. The map $f$ is a continuous single-valued mapping on $X$. We shall construct an almost perfect representation of $f$ and compute its homology.

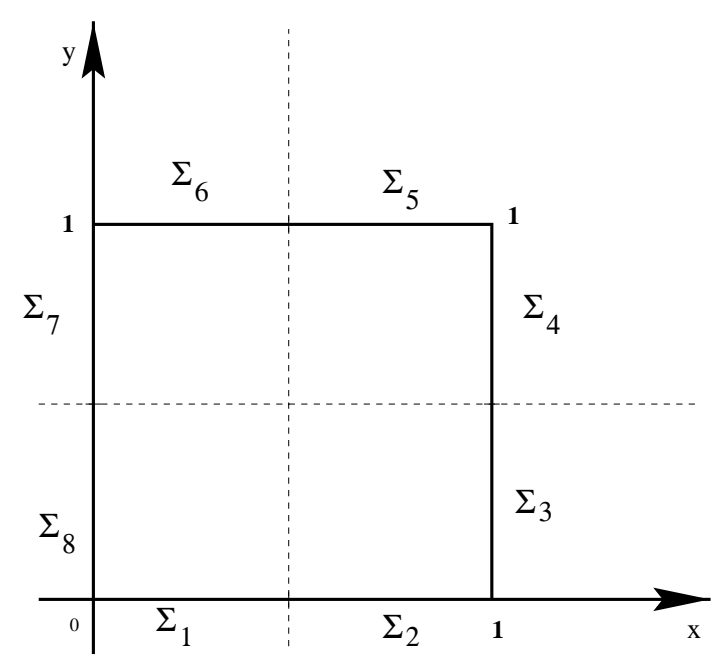

FIGURE 2. Almost perfect representation 
Consider the cubic grid $\mathcal{E}_{k}$ of mesh $\frac{1}{k}\left(k \in \mathbb{N}^{*}\right)$ in $\mathbb{R}^{2}$ whose cells are given by the formula

$$
\begin{gathered}
e_{k}=\frac{1}{k}(x+Q), \quad \text { where } x \in \mathbb{Z}^{2}, \\
\left.Q=I_{1} \times I_{2} \quad \text { and } \quad I_{j}=\right] 0,1[,\{0\} \text { or }\{1\} .
\end{gathered}
$$

Obviously, $X$ is a representable set over $\mathcal{E}_{k}$. It is easily seen that the choice of $\mathcal{E}_{1}$ as a grid will not permit the construction of an almost perfect representation of $f$ with values in $X$. However, that becomes possible for $\mathcal{E}_{k}$ with $k \geq 2$. Indeed, let $k=2$. We define $F$ on the primitive cells, i.e. intervals $\Sigma_{i}$ indicated on Figure 2 by

$$
\begin{array}{lccl}
F\left(\Sigma_{1}\right)=[0,1] \times\{0\}, & F\left(\Sigma_{2}\right)=\{1\} \times[0,1], & F\left(\Sigma_{3}\right)=[0,1] \times\{1\}, \\
F\left(\Sigma_{4}\right)=\{0\} \times[0,1], & F\left(\Sigma_{5}\right)=[0,1] \times\{0\}, & F\left(\Sigma_{6}\right)=\{1\} \times[0,1], \\
F\left(\Sigma_{7}\right)=[0,1] \times\{0\}, & \text { and } & F\left(\Sigma_{8}\right)=\{0\} \times[0,1] .
\end{array}
$$

It follows immediately that $F$ has convex closed and nonempty values on primitive cells and $F$ is star-shaped on vertices (the vertices are the only proper faces of the primitive cells). Indeed, it is easily seen that

$$
F(0,0)=([0,1] \times\{0\}) \cup(\{0\} \times[0,1]),
$$

and we can do the same work for each vertex.

We now define a chain map $\varphi$ induced by $F$. First, on vertices, $\varphi(0,0)=(0,0)$ since $\mathcal{A}(0,0)=([0,1] \times\{0\}) \cap(\{0\} \times[0,1])=\{(0,0)\}$.

By the same argument,

$$
\begin{gathered}
\varphi\left(\frac{1}{2}, 0\right)=(1,0), \quad \varphi(1,0)=(1,1), \quad \varphi\left(1, \frac{1}{2}\right)=(0,1), \quad \varphi(1,1)=(0,0), \\
\varphi\left(\frac{1}{2}, 1\right)=(1,0), \quad \varphi(0,1)=(1,1) \quad \text { and } \quad \varphi\left(0, \frac{1}{2}\right)=(0,1) .
\end{gathered}
$$

On cells of dimension 1, $\varphi$ is computed as indicated below: For example, since

$$
\partial \varphi\left(\Sigma_{1}\right)=\varphi\left(\frac{1}{2}, 0\right)-\varphi(0,0)=\partial\left(\Sigma_{1}+\Sigma_{2}\right) ;
$$

therefore $\varphi\left(\Sigma_{1}\right):=\Sigma_{1}+\Sigma_{2}$, and similarly on the remaining cells. $H_{1}(X)$ is generated by the cycle

$$
\Sigma=\Sigma_{1}+\Sigma_{2}+\ldots+\Sigma_{8}
$$

We get $\varphi_{1}(\Sigma)=2 \Sigma$, hence

$$
H_{1}(f)=\left(\varphi_{1}\right)_{*}=2 I d_{H_{1}(X)} .
$$

Example 6.2. In this example we illustrate the concept of a perfect map by constructing a perfect representation of the identity map $f(x)=x$ on the set $X=\partial\left([0,1]^{2}\right)$. Figure 3 shows the set $Z=\operatorname{st}_{\mathcal{E}_{3}} X$ discussed in Section 5 .

Here, the perfect representation $F$ of $f$ is given as follows:

$$
F: X \longrightarrow \text { st }_{\mathcal{E}_{3}} X \text {. }
$$

On vertices,

$$
F\left(V_{0}\right)=\overline{\Sigma_{1}},\left(V_{1}\right)=\overline{\Sigma_{1}} \cup \overline{\Sigma_{2}}, F\left(V_{2}\right)=\overline{\Sigma_{2}} \cup \overline{\Sigma_{3}}, F\left(V_{3}\right)=\overline{\Sigma_{3}}, \ldots
$$




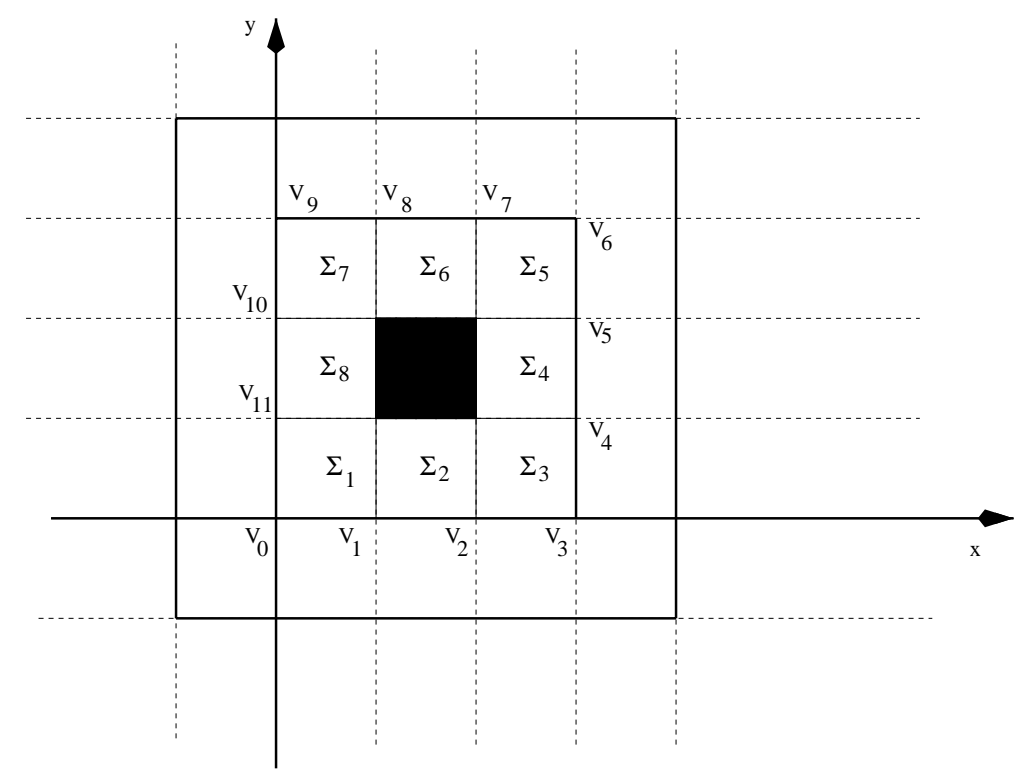

Figure 3. Perfect representation

The vertices $V_{i}$ and the squares $\Sigma_{i}$ are indicated on Figure 3 One then can compute the values of $F$ on the cells of dimension one. For example

$$
F(] V_{0}, V_{1}[)=\overline{\Sigma_{1}} \cap\left(\overline{\Sigma_{1}} \cup \overline{\Sigma_{2}}\right)=\overline{\Sigma_{1}} .
$$

It is easily verified that for each cell $e \in \mathcal{E}_{3}(X)$, we have the inclusion $e \subset \mathcal{A}(e)$. Thus the chain map $\varphi: C(X) \longrightarrow C\left(\operatorname{st}_{\mathcal{E}_{3}} X\right)$ defined by $\varphi(e)=e$ is a chain map induced by $F$. Then, $\varphi_{*}=I d_{H(X)}$.

\section{REFERENCES}

1. M. Allili, Une approche algorithmique pour le calcul de l'homologie de fonctions continues, Ph.D. thesis, Département de mathématique et d'informatique, Université de Sherbrooke, January 1999.

2. E. Begle, The Vietoris mapping theorem for bicompact spaces, Ann. Math., 51 (1950), 534543. MR 11:677b

3. C. A. Delfinado, H. Edelsbrunner, An incremental algorithm for Betti numbers of simplicial complexes on the 3-sphere, Computer Aided Geometric Design, 12 (1995), 771-784. MR 96h:55004

4. T. K. Dey, S. Guha, Algorithms for manifolds and simplicial complexes in Euclidean 3-space, In Proc. 28th ACM Sympos. Theory Comput. (1996), 398-407. CMP 97:06

5. T. K. Dey, H. Edelsbrunner, S. Guha, Computational Topology, Advances in discrete and computational geometry (South Hadley, MA, 1996), 109-143. CMP 99:05

6. D. Dold, Lectures on algebraic topology, Springer-Verlag, Berlin-Heidelberg-New York, (1972). MR 96c:55001

7. R. Ehrenborg, G. Hetyei, Generalizations of Baxter's Theorem and Cubical Homology, J. Combinatorial Theory, Series A, 69 (1995), 233-287. MR 96k:05041]

8. L. Górniewicz, Homological methods in fixed point theory of multi-valued map, Dissertationes Math. (Rozprawy Mat.) 129 (1976), 1-71. MR 52:15438

9. C. S. Iliopoulos, Worst case complexity bounds on algorithms for computing the canonical structure of finite abelian groups and Hermite and Smith normal forms of an integer matrix, SIAM J. Computing 18 (1989), 658-669. MR 91a:20065 
10. T. Kaczynski, M. Mrozek, Conley index for discrete multi-valued dynamical systems, Topology Appl. 65 (1995), 83-96. MR 97d:54066

11. L Stable index pairs for discrete dynamical systems, Canad. Math. Bull. 40 (1997), 448-455. MR 99g:54038

12. T. Kaczynski, M. Mrozek, M. Ślusarek, Homology computation by reduction of chain complexes, Comput. Math. Appl. 33 (1998), 59-70. MR 99a:18001

13. T. Kaczynski, H. Karloff, K. Mischaikow, M. Mrozek, Introduction to Algebraic Topology: A Computational Perspective, book in progress.

14. A. Lecki, Z. Szafraniec, An algebraic method for calculating the topological degree, Banach Center Publ. Vol. 35, Warsaw 1996, 73-83. MR 98b:55002

15. W. S. Massey, A basic course in algebraic topology, Springer-Verlag, New York (1991). MR 92c:55001

16. K. Mischaikow, Conley Index Theory, Dynamical Systems: Montecatini Terme 1994, eds. L. Arnold and C. Jones, Lecture Notes in Math. No. 1609, Springer 1995. MR 97a:58109

17. K. Mischaikow, M. Mrozek, A. Szymczak, J. Reiss, From time series to symbolic dynamics: An algebraic topological approach, preliminary version.

18. K. Mischaikow, M. Mrozek, Chaos in the Lorenz equations: a computer assisted proof, Bull. Amer. Math. Soc. (N.S.) 32 (1995), 66-72. MR 95e:58121

19. K. Mischaikow, M. Mrozek, A. Szymczak, Chaos in the Lorenz equations: a computer assisted proof, Part III: the classical case. Preprint.

20. M. Mrozek, Topological invariants, multivalued maps and computer assisted proofs in dynamics, Computers and Mathematics 32 (1996), 83-104. MR 97h:58144

21. J. R. Munkers, Elements of algebraic topology, Addison-Wesley (1984). MR 85m:55001

22. H. W. Siegberg, G. Skordev, Fixed point index and chain approximations, Pacific Journal of Mathematics 102, No. 2 (1982), 455-486. MR 84d:55003

23. A. Szymczak, A combinatorial procedure for finding isolating neighbourhoods and index pairs, Proc. of the Royal Soc. of Edinburgh 127 A (1997), 1075-1088. MR 98i:58151

24. P. Zgliczynski, Fixed points for iterations of maps, topological horseshoe and chaos, Top. Meth. in Nonlin. Anal. 8 (1996), 169-177. MR 98m:58106

25. - Computer assisted proofs of chaos in the Rössler equations and the Hénon map, Nonlinearity 10 (1997), 243-252. MR 98g:58120

Center for Dynamical Systems and Nonlinear Studies, School of Mathematics, Georgia Institute of Technology, Atlanta, Georgia 30332-0160

E-mail address: allili@math.gatech.edu

Département de Mathématiques et d'Informatique, Université de Sherbrooke, SherBRoOKe, QuÉBEC J1K 2R1, CANADA

E-mail address: kaczyn@dmi.usherb.ca 